\title{
Identification of Quadriceps-Shank Dynamics Using Randomized Interpulse Interval Stimulation
}

\author{
Henry M. Franken, Student Member, IEEE, Peter H. Veltink, Member, IEEE, Roel Tijsmans, \\ Henk Nijmeijer, Senior Member, IEEE, and Herman B. K. Boom
}

\begin{abstract}
Model structures for artificially stimulated paralyzed muscle-limb system dynamics were developed and experimentally evaluated in paraplegic patients. The examined system consisted of the quadriceps, electrically stimulated using surface electrodes, and a freely swinging shank. The interpulse interval of the stimulation sequence was randomized to obtain persistent system excitation. The outputs of the system were the angular position, velocity, and acceleration, measured by externally mounted sensors. This paper especially reports on model identification of the active quadriceps dynamics and the angle prediction performance of the total quadriceps-shank model. Second-order modeling of the twitch dynamics with delay did not significantly improve the prediction results in comparison to a zero-order model with delay $(\alpha=0.05)$. Nonlinear torque-angle and torque-angular velocity relations in combination with a zero-order model (with delay) only slightly improved the prediction results for large prediction intervals $(\alpha=0.05$ ). The delay between stimulation input and resulting knee joint acceleration appeared to be joint angle dependent and was estimated to be largest in the knee angle range near knee extension, i.e., when quadriceps muscle is shortest.
\end{abstract}

\section{NOMENCLATURE}

$a_{1}, a_{2} \quad$ Coefficients of the autoregressive part of model 2 .

$b_{1}, b_{2} \quad$ Coefficients of the moving average part of model 2.

$c_{1}, c_{2} \quad$ Coefficients of the whitening filter.

$d \quad$ Number of delay steps.

$f(\varphi) \quad$ Torque-angle relationship.

$g(\dot{\varphi}) \quad$ Torque-angular velocity relationship.

$k \quad$ Time step index.

$k_{1}, k_{2} \quad$ Parameters of the passive elasticity function.

$k_{1} \quad$ Elasticity parameter $k_{1}$ divided by the inertia.

$p \quad$ Number of prediction steps.

$q \quad$ Delay operator.

$u_{k} \quad$ Input recruitment level at time step $k$

$\varphi_{k} \quad$ Knee joint angle at time step $k$.

$\hat{\varphi}_{k} \quad$ Estimated knee joint angle for time step $k$.

$\dot{\varphi}_{k} \quad$ Knee joint angular velocity at time step $k$.

Manuscript received February 10, 1994; revised February 21, 1995. This work was partially supported by the European Fund for Regional Development.

H. M. Franken, P. H. Veltink, R. Tijsmans, and H. B. K. Boom are with the Department of Electrical Engineering, University of Twente, $7500 \mathrm{AE}$ Enschede, The Netherlands.

H. Nijmeijer is with the Department of Applied Mathematics, University of Twente, $7500 \mathrm{AE}$ Enschede, The Netherlands.

IEEE Log Number 9410754. $\ddot{\varphi}_{k} \quad$ Knee joint angular acceleration at time step $k$.

$\gamma_{\text {angle }} \quad$ Parameter of $f(\varphi)$.

$\gamma_{\text {velocity }}$ Parameter of $g(\dot{\varphi})$.

$A(q) \quad$ Autoregressive part of activation dynamics.

$B(q) \quad$ Moving average part of activation dynamics.

$I \quad$ Inertia of the shank.

$D \quad$ Damping of the passive system $\left(D^{\prime}=D / I\right)$.

$F(\varphi) \quad$ Static components of passive system.

$G \quad$ Gravity component of the system $\left(G^{\prime}=G / I\right)$.

$G_{1} \quad$ Gain parameter of model $1\left(G_{1}^{\prime}=G / I\right)$.

$G_{2} \quad$ Gain parameter of model $3\left(G_{2}^{\prime}=G / I\right)$.

$J_{\varphi, p} \quad$ Evaluation criterion for the performance of the models (12).

$M_{a, k} \quad$ State vector of the activation dynamics.

$M_{k} \quad$ Active knee joint torque at time step $k$.

$N \quad$ Degree of polynomials of DARMA model.

$\hat{T} \quad$ Estimated integrated equivalent acceleration response to a stimulus.

$T_{s} \quad$ Sampling period.

\section{INTRODUCTION}

$\mathbf{T}$ HE RESTORATION of functional tasks to paralyzed muscle-limb systems by means of functional electrical stimulation (FES) implies a challenging control problem. Nonlinear passive characteristics of the limbs, nonlinear dynamics [12], and significant time variation (e.g., due to rapid fatigue [7], [15], [30] of the stimulated muscles) require an approach based on knowledge of the system [17]. Thus, the control performance of electrically stimulated paralyzed human limbs could be improved if an adequate model for this system would be available [5], [10]-[12], [14], [16], [20], [28], [40], [44]-[46]. It should facilitate model-based control techniques, such as feed-forward and predictive control, which generally advance the control of nonlinear time-varying systems [31].

Muscle dynamic behavior has been described in terms of various model types. Hill [21] proposed a mechanical analog, including a contractile element with force-velocity dependency in series with a nonlinear elastic element. Various authors have extended Hill's model for conditions not dealt with in the original study, such as eccentric contractions and isometric force-length dependencies [25]-[27], [36], [38], [39], [49]. Various more complex model structures were proposed by Huxley [24], Zahalak [51], and Hatze [19], including mathematical descriptions of known biophysical processes. 
Model-based control techniques, such as feed-forward and predictive control strategies, impose certain requirements on models of muscle-joint systems. The model should evidently be sufficiently accurate. Its parameters must be identifiable using FES as excitation signal. Additionally, the number of model parameters that have to be tracked to account for taskrelated fatigue-induced time-variation in the system must be small [31]. The more complex model structures [19], [24], [51] contain many parameters which are not identifiable using FES as excitation signal, and are therefore not suitable for modelbased control. Hill-based models seem most appropriate for the design of control strategies for electrically stimulated muscle, even linearized models have been reported to be sufficiently accurate within restricted operating conditions [3]-[5], [32], [42], [48], [52]. Animal studies have shown that modelbased control of FES-induced muscle-joint movements using Hill-based muscle models in combination with passive limb dynamics improved the control performance significantly in comparison to a classical (PID) control scheme [45].

Different control tasks may require different stimulation electrodes characteristics: Relatively slow position control of a low inertia system (upper extremity applications) requires a good modulation of recruitment, which can only be achieved with intramuscular [11], [29] or neural electrodes [45], but not with surface electrodes. In contrast, control of relatively fast movements of large inertia systems (lower extremity control during gait) requires a less precise recruitment control, since it requires large mechanical impulses which can only be achieved at maximal recruitment. In these cases, the number and timing of the stimulation pulses are especially important [44], which may be sufficiently achievable using surface electrodes. However, intramuscular or neural electrodes are also preferable in lower extremity applications because they can more selectively and more efficiently stimulate the required muscles [33].

We have currently studied the paralyzed quadriceps-shank system to gain insight in the feasibilities of model-based predictive position control of electrically stimulated paralyzed human limbs using surface electrodes [16], [44], [46]. Modelbased predictive control is especially important in real-time position control in the presence of relatively large inertias and low maximal muscle torque saturation, like in the control of lower limb movement during gait [44]. Without a model of the system other strategies can be used without real-time feedback, for example, open loop application of stimulation patterns obtained by experimental optimization [34] or cycleto-cycle adaptation of stimulation patterns [44]. Real-time position control of relatively slow movements of the upper limbs (relatively small inertias) is feasible without the need of model-based predictions of the system behavior [11], [29].

Several authors have already reported on model-based control of the paralyzed quadriceps-shank system (some in combination with hamstring stimulation). Hausdorff and Durfee [20] described an open-loop position control of the knee joint using surface electrical stimulation of the quadriceps and hamstrings. They developed an inverse model consisting of load dynamics (i.e., passive characteristics of the knee joint and shank), linear muscle activation dynamics, (static) recruitment characteristics, and (static) isometric torque-angle dependency. Open-loop prefiltering of the stimulation signals on the basis of this inverse model improved open-loop tracking of joint position. However, Hausdorff and Durfee [20] did not report the predictive capabilities of the model while the knee joint tracking performance was still poor. Hatwell et al. [18] developed a model reference adaptive controller for surface electrical stimulation of quadriceps to track a prescribed knee joint trajectory in paraplegics. The quadriceps model consisted of a nonlinear part followed by a linear deterministic Auto Regressive Moving Average (ARMA) model. Controller performance was only satisfactory when the model reference parameters were chosen so as to severly limit the closed-loop bandwidth. Knee joint movements with a cycle time approaching the natural frequency of the passive system, which are common in natural gait, could not be controlled. Also, frequent clamping of the control signal impeded validation of the reported models from the displayed registrations. Up to now, it has not been shown that any of the quadriceps-shank models used for controller tuning and adaption, as described above, accurately predict the state of this human muscle-limb system in an FES-induced dynamic contraction.

The current paper reports on the development and evaluation of model structures for muscle-limb systems, describing the responses to single stimulation pulses. Fused responses to stimulation bursts with varying interpulse interval times may be considered as a summation of twitch responses, although the summation may not be linear [5], [6], [9]. We used the stimulation-joint movement (input-output) data from artificially stimulated quadriceps-shank system in paraplegic subjects to investigate the identification and predictive capabilities of Hill-based muscle-limb models. This was done in the knee angle range of -40 to $60^{\circ}$ (with the knee angle $\varphi$ taken as zero when the shank is at rest). This operating range corresponds to the range used in our previous experimental study of cycle-to-cycle control [44] and our current study on model-based predictive control. It does not, however, correspond to the knee angle range used during gait. However, the chosen system and operating range can be considered as a simple example system, which allows the study of dynamic models and model-based control, of which the principles may be applicable in the control of knee and hip movements during gait. The identification of the passive part of the system, i.e., passive knee joint and shank dynamics, has been reported in detail in [16]. The current paper focuses on the identification of the active system (quadriceps muscle) and predictive capabilities of the total model for the quadriceps-shank system so as to serve for feed-forward or predictive control techniques.

\section{THEORY}

\section{A. Model Structures}

The quadriceps-shank system was considered. The shank was moving freely in the range of -40 to $60^{\circ}$ (with the 


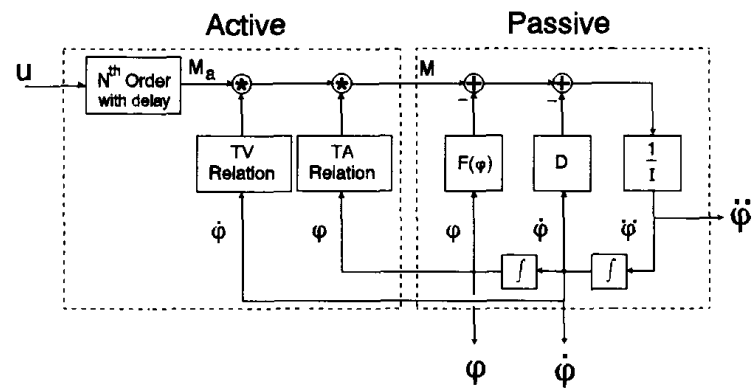

Fig. 1. Block diagram of the considered quadriceps-shank system (see (1)-(7)). Input to the system is stimulation $u$ (with $0 \leq u \leq 1$ ). The muscle dynamics consist of activation dynamics ( $N$ th order with delay), modulated by a torque-knee angle (TA) relation and a torque-knee angular velocity (TV) relation. The passive system is described by damping (D), passive elasticities, and gravity (grouped in $F(\varphi)$ ), and inertial properties (I). These parameters of the passive system were assumed to be independent of the active quadriceps torque (M).

knee angle $\varphi$ taken zero when the shank is at rest), while the positions of the knee joint and upper limb were fixed (the subjects were sitting).

The musculoskeletal-load model structure (Fig. 1) consisted of activation dynamics, multiplied by a static knee angle and a knee angular velocity dependency (active system), as well as passive properties of the knee joint and shank (passive system). Veltink et al. [45] and Shue et al. [42] identified similar model structures for the musculoskeletal-load system in animal studies.

1) Active System: The muscle model (Fig. 2) consisted of a Discrete-time Auto Regressive Moving Average (DARMA) description of the activation dynamics (1), multiplied by a static nonlinearity (2), yielding the active knee torque. This model structure is generally refered to as a Wiener system [24]

$$
\begin{aligned}
A(q) \cdot M_{a, k} & =B(q) \cdot u_{k-d} \\
M_{k} & =M_{a, k} \cdot f\left(\varphi_{k}\right) \cdot g\left(\dot{\varphi}_{k}\right)
\end{aligned}
$$

in which $A$ and $B$ are polynomials of degree $N$ in $q$ (delay operator) [1], [31]; $M_{a, k}$ is the state vector of the activation dynamics; $k$ is the time step (equal to the sampling period); $u_{k}$ is the recruitment level, defined as the steady-state knee torque generated by the stimulation at a fixed knee angle divided by the maximally generated knee torque obtained at saturation at this knee angle [13], [15] (thus $0 \leq u_{k} \leq 1$ ); $d$ is the number of delay steps from input $u$ and to output $M_{a, k} ; M_{k}$ is the active quadriceps torque at the knee joint; $\varphi_{k}$ and $\dot{\varphi}_{k}$ are the knee angle and angular velocity, respectively; $f$ is the static quadriceps torque-muscle length dependency; and $g$ is the static quadriceps muscle contraction velocity dependency.

The muscle length and contraction velocity dependencies are the basic components of Hill-based muscle models. They were expressed in a knee angle and knee angular velocity dependency, respectively. Control of limbs is usually expressed in joint space. Thus, it is advantageous to formulate length and velocity dependency of the muscle in terms of joint angle and velocity [47]. In the currrent paper, following from its definition, the recruitment level $u_{k} \in\{0,0.5,1\}$.
Analyzing three special cases of (1)-(2) provided insight in the complexity necessary for accurate joint state prediction

$$
\begin{aligned}
\operatorname{model~1:~} \quad M_{k}= & G_{1} \cdot u_{k-d} \\
\operatorname{model~2:~} \quad M_{a, k}= & -a_{1} \cdot M_{a, k-1}-a_{2} \cdot M_{a, k-2} \\
& +b_{1} \cdot u_{k-1-d}+b_{2} \cdot u_{k-2-d} \\
& \quad \text { with } M_{k}=M_{a, k} \\
\text { model 3: } \quad M_{k}= & G_{2} \cdot u_{k-d} \cdot\left(1+\gamma_{\text {angle }} \cdot \varphi_{k}\right) \\
& \cdot\left(1+\gamma_{\text {velocity }} \cdot \dot{\varphi}_{k}\right) .
\end{aligned}
$$

Model 1 is a simple stateless zero-order transfer function, whereas model 2 is a second-order DARMA model [31] (see also [2]-[5], [45], [48]). Thus, neither model 1 nor model 2 contained static nonlinearities. In model 3 , we investigated whether such a nonlinear relation for the combined torqueangle and torque-angular velocity dependency could improve the prediction capability of the model (see also [42], [45]).

The discrete-time model structure for the active system allowed a simple formulation of system delay. Furthermore, identification theory for discrete-time systems with delay is widely available in comparison to continuous-time systems with delay [31].

2) Passive System: The model structure for the passive system (Fig. 2) was taken directly from [16]. The model

$$
\begin{aligned}
M & =I \cdot \ddot{\varphi}+D \cdot \dot{\varphi}+F(\varphi) \\
F(\varphi) & =G \cdot \sin (\varphi)-k_{1} \cdot\left(\exp \left(-k_{2} \cdot \varphi\right)-1\right)
\end{aligned}
$$

formulates in continuous time, with the advantage that each parameter is interpretable from the underlying biomechanics, which are inertial properties $(I)$ of the shank, damping $(D)$ in the knee joint, a gravitation component $(G)$, and passive elasticity (exponential term) in the knee joint. The contribution of elasticity in the chosen operating range was only significant in knee flexion and was therefore modeled by the exponential term in (7) [16]. $M$ is the active torque at the knee joint. The equivalent sampled signal is $M_{k}$.

\section{METHODS}

\section{A. Subjects}

Three spinal cord injured patients (JM: T8 level complete, TN: T5-6 level complete, and OT: T9-10 level complete) participated in this study. Additional information concerning the subjects can be found in [16].

\section{B. Experimental Set-Up}

The experimental set-up used in the current study has been described in detail and characterized in [16], where it served to identify the passive system $((6)$ and (7)). In short, the subjects were seated on a chair allowing the shank to swing freely. The quadriceps were stimulated using surface electrodes. An externally mounted goniometer measured the knee angle $(\varphi)$. The knee angular velocity $(\dot{\varphi})$ was derived off-line from $\varphi$ 


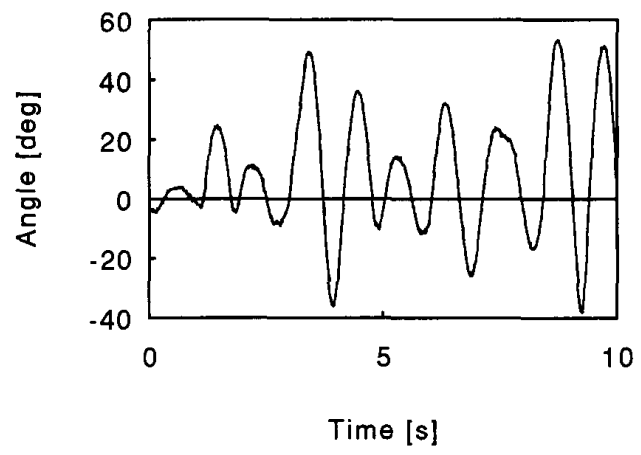

(a)

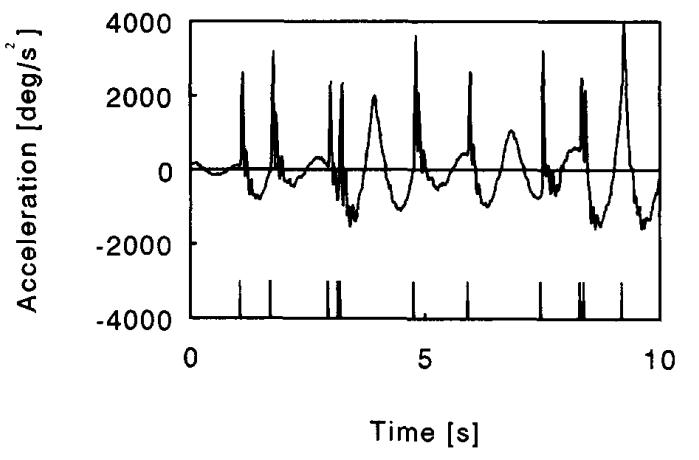

(c)

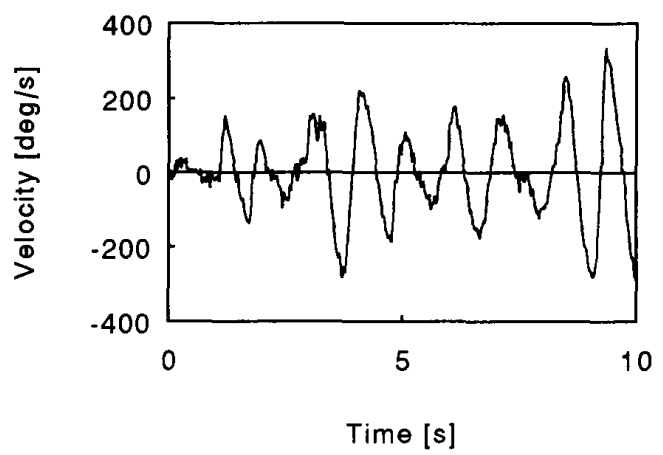

(b)
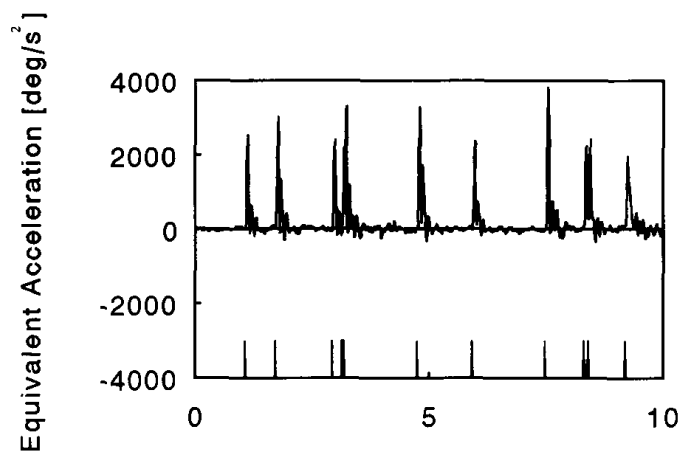

Time [s]

(d)

Fig. 2. Measured and estimated signals of the freely swinging shank system. The quadriceps were stimulated with a pseudorandom IPI stimulation sequence at maximal recruitment. The displayed sequence was cut out of pseudorandom trial 1 on subject TN-right which was used for the model identification. (a) The measured knee angle. (b) Knee angular velocity estimated from the angle signal. (c) The measured angular acceleration and applied stimulation pulses. (d) The estimated equivalent acceleration $M / I$ due to quadriceps stimulation. $M / I$ was obtained by subtracting the contributions of the passive system from the measured angular acceleration. Each sample of $M / I$ resulted from substitution of measured knee angle, angular velocity, and acceleration in the identified model for the passive system (8). The stimulation pulses are also shown.

and the knee angular acceleration $(\ddot{\varphi})$ was measured using accelerometers.

1) Stimulation and Data Recording: Stimulation consisted of applying rectangular monophasic current pulses. Recruitment level $\left(u_{k}\right)$ was modulated by varying the pulse amplitude with fixed pulse width $(300 \mu \mathrm{s})$. Maximal and half-recruitment represented two recruitment levels for which the pulse amplitudes were estimated on the basis of an isometric recruitment curve [15], [16]. Stimulation pulses were synchronized with the beginning of a sampling period $\left(T_{s}=10 \mathrm{~ms}\right)$, yielding an interpulse interval (IPI) quantization of $T_{s}$. The signals from both the goniometer and the accelerometers, as well as the applied stimulation, were likewise obtained at the beginning of the sampling period. Data analysis was off-line.

\section{Protocol}

We successively applied three different pseudorandom IPI stimulation sequences of $5 \mathrm{~min}$ each to the quadriceps muscle with a rest period of $15 \mathrm{~min}$ in between trials. A twopart probability density function [22] determined the IPI's per sequence, ensuring sufficient excitation of the active and passive system: In our previous paper [16], we showed that this distribution resulted in the activation of the muscle in the whole desired operating range of the passive system, defined as the state space of knee angle and angular velocity covered by a passive swing of the shank. The recruitment levels of the stimulation pulses in trials 1 and 2 were fixed at $u_{k}=1$. In trial $3, u_{k}=0.5$.

\section{Identification Method}

Equations (6) and (7), divided by the inertia $I$ for identification purposes [16], read

$$
M / I=\ddot{\varphi}+D^{\prime} \cdot \dot{\varphi}+G^{\prime} \cdot \sin (\varphi)-k_{1}^{\prime} \cdot\left(\exp \left(-k_{2} \cdot \varphi\right)-1\right)
$$

with the prime indicating division of the parameters by the inertia $I . M / I$ is active torque divided by the inertia $I$ of the shank, assuming a fixed value of inertia, and will be called equivalent acceleration. $M / I$ can be estimated from the measured acceleration by subtracting the contribution due to the passive system. The identification method and results of the passive system have already been reported in detail [16]. In the present paper, the parameters of the three muscle models $((3)-(5))$ were identified using (only) the knee joint signals $(\varphi, \dot{\varphi}, \ddot{\varphi})$ and the computed equivalent acceleration $M / I$ as 
obtained during pseudorandom stimulation trial 1. Noteworthy, the time step of the discrete models ((3)-(5)) was equal to the sampling period $T_{s}$. Thus, a stimulation pulse at time step $k=n$ in trial 1 (maximal recruitment) resulted in $u_{n}=1$.

1) Data Analysis: From trial 1, epochs of $\left[\begin{array}{lll}\varphi & \dot{\varphi} & \ddot{\varphi}\end{array}\right.$ $\left.\left(M_{k} / I\right) u_{k}\right]$ were extracted during which isolated twitches were recorded. Each lasted the assumed twitch duration (of $250 \mathrm{~ms}$, i.e., 25 samples) and started with a stimulation pulse. A period of no stimulation, equal to the twitch duration, preceded and succeeded an isolated twitch.

\section{2) Identification:}

a) Model 1: From (3), considering $G_{1}^{\prime}=G_{1} / I$ being a constant

$$
G_{1}^{\prime}=\sum_{k=1}^{L}\left(M_{k} / I\right) / \sum_{i=1}^{L} u_{i}
$$

where $L$ is the total number of samples of all selected epoches. Thus, $G_{1}^{\prime}$ equals the average equivalent acceleration impulse due to a stimulation pulse.

b) Model 2: A generalized least square algorithm [31] minimized the mean square error between predicted and actual equivalent accelerations $\hat{M}_{k} / I$ and $M_{k} / I$, as obtained from (8), employing all extracted epochs

$$
\begin{aligned}
\left(\hat{M}_{k} / I\right)= & -a_{1} \cdot\left(\hat{M}_{k-1} / I\right)-a_{2} \cdot\left(\hat{M}_{k-2} / I\right)+b_{1}^{\prime} \cdot u_{k-1-d} \\
& +b_{2}^{\prime} \cdot u_{k-2-d}+e_{k}+c_{1} \cdot e_{k-1}+c_{k-2} \cdot e_{k-2}
\end{aligned}
$$

with the prime again indicating division of the parameters by the inertia $I$ of the shank. The last three terms served as a whitening filter on the residual error $e(k)$. It allowed a description of disturbances [31], such as due to the nonrigid attachment of the accelerometers to the shank [16], [35], but was was not used in the evaluation simulations. The smallest residue error for $\forall d \in[0 . .10]$ provided the number of delay steps $d$ for all three models [31].

c) Model 3: Parameters of the static nonlinear relation for the combined torque-angle and torque-angular velocity dependencies were identified from the integrated individual twitch responses, integrating $M / I$ over $250 \mathrm{~ms}$. Also, the knee angle and angular velocity were extracted at $d$ steps after the beginning of each twitch, i.e., $\varphi_{k+d}$ and $\dot{\varphi}_{k+d}$. The resulting set of data was used to identify the parameters of (5)

$$
\hat{T}=G_{2}^{\prime} \cdot\left(1+\gamma_{\text {angle }} \cdot \varphi\right) \cdot\left(1+\gamma_{\text {velocity }} \cdot \dot{\varphi}\right) .
$$

The Levenburg-Marquardt algorithm [31] iteratively fitted the parameters $G_{2}^{\prime}=G_{2} / I, \gamma_{\text {angle }}$ and $\gamma_{\text {velocity }}$ by minimizing the mean square error between the estimated integrated equivalent acceleration response to a stimulus, called $\hat{T}$, and the actual integrated response $\Sigma M / I$ obtained from the measured equivalent acceleration.

\section{E. Evaluation of Model Prediction Capability}

Model evaluation was performed on all three pseudorandom stimulation trials. The following root mean square ( $\mathrm{rms}$ ) criterion was used to assess the models' prediction capability:

$$
J_{\varphi, p}=\sqrt{\frac{1}{l} \cdot \sum_{i=1+p}^{l+p}\left[\varphi_{i}-\hat{\varphi}_{i}\right]^{2}}
$$

in which $l$ is the number of times a prediction was performed in the considered trial and $p$ the prediction interval. The measured knee angle at time $k=i$ is $\varphi_{i}$ and the knee angle predicted for time $k=i$ is $\hat{\varphi}_{i}$.

$J_{\varphi, p}$ was computed at four different prediction intervals $(100,250,500 \mathrm{~ms}$, and $1 \mathrm{~s}$; i.e., corresponding to $p=10$, 25,50 , or 100 samples, respectively) using the three identified models for the active system (3)-(5) and the same passive system. The state measured at time step $k=n\left(\varphi_{n}, \dot{\varphi}_{n}\right)$, with $n=1 \cdots l$, was used to initialize the passive part of the model, while the active part was initialized on the basis of the previous simulation run. A stimulation pulse at time step $k=m$ at maximal recruitment represented $u_{m}=1$, at halfrecruitment $u_{m}=0.5$, and no stimulation pulse $u_{m}=0$. Then, model simulations were performed for $p$ samples, applying the actual stimulation only. The passive system was integrated forward each sample (time duration $T_{S}$ ) using a fourth-order Runge-Kutta integration routine. Subsequently, the measured knee angle at time $k=i+p$ and the predicted knee angle for time $k=i+p$ were used to evaluate the criterion (12).

1) Statistics: Differences in prediction performance were tested statistically using a nonparametric sign test (see also [15], [16]), with a significance level of $\alpha=0.05$. The values for $J_{\varphi, p}$ for the three models were compared in pairs within a subject per prediction interval for trials 2 and 3 .

\section{RESULTS}

\section{A. Identification of Active System}

Fig. 2 depicts a typical registration of measured and estimated knee joint signals, applied stimulus pulses, and the computed equivalent acceleration $M / I$ (according to (8)) of trial 1. Fig. 2(d) shows that quadriceps activity was only detected during the application of stimulation pulses. There is a small additional ripple and overshoot on the equivalent acceleration $M / I$, which may be caused by the nonrigid attachment of the accelerometers to the shank [16].

1) Model 1: The parameter values of model 1 (3) for all subjects are given in Table I. $G_{1}^{\prime}$ was the largest for subject TN, which corresponds to TN's also exhibiting the largest isometric steady-state knee torque generated at saturation during the recruitment curve. There was a significant difference in $G_{1}^{\prime}$ between subjects, whereas intrasubject difference in $G_{1}^{\prime}$ between the left and right leg was small. $G_{1}^{\prime}$ was estimated with large standard deviation within each leg, indicating that the integrated twitch responses showed significant variance during the trials. This may be largely attributed to the knee angle and angular velocity dependence of the twitch response (see model 3). The estimated delay $d$ (see Table I) differed between subjects. The low resolution in $d$ (units of $T_{s}=10$ ms) complicated an accurate estimation. 
TABLE I

Parameter Values for Impulse $G_{1}^{\prime}$ of Model 1 and the Delay $d$ for the active System of the Participating Subjects. The Delay $d$ Was Taken Such That It YIELded the Smallest Residual ERRor in the Least SQuares Identification of MOdel 2. LeFt OR Right Leg Is Indicated. The Average of $G_{1}^{\prime}$ and $d$ AND the Standard Deviations (StD) ARe Also Given

\begin{tabular}{llll}
\hline subject & $\mathrm{G}_{1}^{\prime}\left[\mathrm{deg} / \mathrm{s}^{2}\right]$ & $(\mathrm{std})$ & delay: $d[\mathrm{~ms}]$ \\
\hline TN-left & $1.46 \mathrm{e} 4$ & $(4.2 \mathrm{e} 3)$ & 40 \\
TN-right & $1.43 \mathrm{e} 4$ & $(4.2 \mathrm{e} 3)$ & 50 \\
JM-left & $7.98 \mathrm{e} 3$ & $(3.3 \mathrm{e} 3)$ & 40 \\
JM-right & $8.00 \mathrm{e} 3$ & $(2.9 \mathrm{e} 3)$ & 50 \\
OT-left & $7.32 \mathrm{e} 3$ & $(1.7 \mathrm{e} 3)$ & 40 \\
\hline average & $1.05 \mathrm{e} 4$ & - & 44 \\
std & $3.7 \mathrm{e} 3$ &.-- & 5.5 \\
\hline
\end{tabular}

TABLE II

Parameter Values for Model 2 for the Active System of the Participating Subjects. Left or Right Leg Is Indicated. The Corresponding Delay Is Given in Table I. The Average of the Parameters with the Standard Deviations (StD) ARe Also Given

\begin{tabular}{lllll}
\hline \hline subject & $a_{1}$ & $a_{2}$ & $b_{1}^{\prime}\left[\mathrm{deg} / \mathrm{s}^{2}\right]$ & $b_{2}^{\prime}\left[\mathrm{deg} / \mathrm{s}^{2}\right]$ \\
\hline TN-left & -1.41 & 0.60 & $5.41 \mathrm{e} 2$ & $6.45 \mathrm{e} 2$ \\
TN-right & -1.46 & 0.60 & $5.14 \mathrm{e} 2$ & $6.14 \mathrm{e} 2$ \\
JM-left & -1.50 & 0.60 & $4.06 \mathrm{e} 2$ & $3.24 \mathrm{e} 2$ \\
JM-right & -1.42 & 0.61 & $5.33 \mathrm{e} 2$ & $6.40 \mathrm{e} 2$ \\
OT-left & -1.49 & 0.55 & $4.22 \mathrm{e} 2$ & $3.28 \mathrm{e} 2$ \\
\hline average & -1.46 & 0.59 & $4.84 \mathrm{e} 2$ & $5.10 \mathrm{e} 2$ \\
std & $4.1 \mathrm{e}-2$ & $2.0 \mathrm{e}-2$ & $6.5 \mathrm{e} 1$ & $1.7 \mathrm{e} 2$ \\
\hline
\end{tabular}

2) Model 2: The parameter values of model 2 (4) for all subjects, as given in Table II, significantly differ between subjects in the parameters $b_{1}^{\prime}$ and $b_{2}^{\prime}$, similarly to $G_{1}^{\prime}$ (see the standard deviations in the average parameter values). This difference is much lower in parameters $a_{1}$ and $a_{2}$. Delay $d$ is given in Table $\mathrm{I}$.

3) Model 3: The parameter values of model 3 (5), iteratively determined using the Levenburg-Marquardt algorithm, are given in Table III. Large intersubject differences were found in all parameters. The rms errors in the estimations of the integrated equivalent acceleration responses to the stimuli $\hat{T}$ were lower in all cases when using model $3(11)$ than when using model $1\left(\hat{T}=G_{1}^{\prime}\right.$ : (9)) (Table IV), indicating an improved estimation of twitch responses when taking the angle and angular velocity dependencies into account. This is illustrated in Figs. 3 and 4. Fig. 3 examplifies the measured integrated twitch responses $\Sigma M / I$ versus the knee angle $\varphi$ or knee angular velocity $\dot{\varphi}$ without taking account of the angle and angular velocity dependencies. Fig. 4 shows the same data after taking account of these dependencies: $\Sigma(M / I) /(1+$
TABLE III

Parameter Values for MOdel 3 for the Active System of the Participating SubJects. LeFt OR Right Leg Is IndiCated. The Corresponding Delay Is Given in Table I. The Average of the Parameters with the Standard Devlations (STD) ARE ALso GIVEN WITH $\varphi$ IN [DEG] AND $\dot{\varphi}$ IN [DEG/S]

\begin{tabular}{llll}
\hline subject & $G_{2}^{\prime}\left[\mathrm{deg} / \mathrm{s}^{2}\right]$ & $\gamma_{\text {angle }}[1 / \mathrm{deg}]$ & $\gamma_{\text {velocity }}[\mathrm{s} / \mathrm{deg}]$ \\
\hline TN-left & $1.67 \mathrm{e} 4$ & $-7.31 \mathrm{e}-3$ & $-1.64 \mathrm{e}-3$ \\
TN-right & $1.55 \mathrm{e} 4$ & $-5.31 \mathrm{e}-3$ & $-2.05 \mathrm{e}-3$ \\
JM-left & $8.67 \mathrm{e} 3$ & $-2.16 \mathrm{e}-2$ & $-1.60 \mathrm{e}-3$ \\
JM-right & $8.46 \mathrm{e} 3$ & $-1.90 \mathrm{e}-2$ & $-1.70 \mathrm{e}-3$ \\
OT-left & $7.74 \mathrm{e} 3$ & $-9.40 \mathrm{e}-3$ & $-2.50 \mathrm{e}-3$ \\
\hline average & $1.14 \mathrm{e} 4$ & $-1.25 \mathrm{e}-2$ & $-1.90 \mathrm{e}-3$ \\
std & $4.2 \mathrm{e} 3$ & $7.3 \mathrm{e}-3$ & $3.8 \mathrm{e}-4$ \\
\hline
\end{tabular}

TABLE IV

RMS ERRORS IN THE ESTIMATION OF THE INTEGRATEd EQUIVALENT ACCEleration Responses to Stimuli $\hat{T}$ Using Model 1 ( $\hat{T}=G_{1}^{\prime}$ : (9)) ANd MOdel 3 (11). THE RMS ERrors Were Determined by Comparing the Estimated Responses With the Measured Responses of Trials 1 (Identification Trials). The Rms Errors are Given as Percentages of the Average Integrated Equivalent Acceleration $G_{1}^{\prime}$

\begin{tabular}{lcc}
\hline subject & $\begin{array}{c}\text { \% RMS error in estimated integrated twitch response } \\
\text { model } 1\end{array}$ & $\begin{array}{c}\text { model 3 } \\
\text { TN-left }\end{array}$ \\
TN-right & $30 \%$ & $20 \%$ \\
JM-left & $34 \%$ & $21 \%$ \\
JM-right & $48 \%$ & $12 \%$ \\
OT-left & $32 \%$ & $29 \%$ \\
\hline
\end{tabular}

$\left.\gamma_{\text {velocity }} \dot{\varphi}\right)$ versus $\varphi$ (Fig. 4(a)) and $\Sigma(M / I) /\left(1+\gamma_{\text {angle }} \varphi\right)$ versus $\dot{\varphi}$ (Fig. 4(b)) are displayed. The delay $d$ is given in Table I.

\section{B. Model Evaluation Representation}

Figs. 5 and 6 display typical prediction results of the knee angle at two different prediction intervals (100 and $1000 \mathrm{~ms})$, taken out of trial 2 which was not used for the identification.

The performance of the models identified was quantitatively estimated by inspecting the values for the evaluation criterion $J_{\varphi, p}$ at the different prediction intervals for all trials (summarized in Table V).

We statistically evaluated the prediction performances at trials 2 (full recruitment: $u_{k}=1.0$ ) and 3 (half recruitment: $u_{k}=0.5$ ). These trials were not used for the identification of the models. For both trials, small prediction intervals (100 and $250 \mathrm{~ms}$ ) yielded small values of $J_{\varphi, p}$, which were not significantly different between all three models $(\alpha=0.05)$. Even at the largest prediction interval $(1000 \mathrm{~ms})$, the prediction errors were not significantly different between models 1 and 2 $(\alpha=0.05)$. However, model 3 performed significantly better 


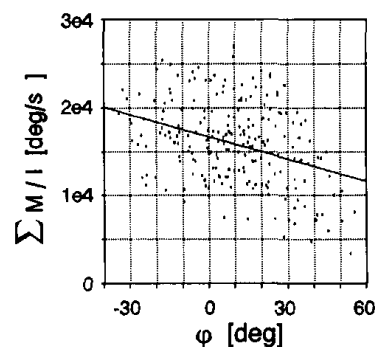

(a)

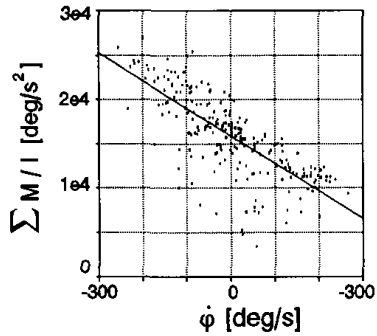

(b)
Fig. 3. Integrated twitch responses $\Sigma M / I$ versus the corresponding knee angle $\varphi($ a) and the knee angular velocity $\dot{\varphi}$ (b) at $d$ steps after the stimulation pulse (in patient TN-left; trial 1 ). The angle and angular velocity dependencies of the twitch response were not taken into account (model 1). Linear regression lines are also shown.

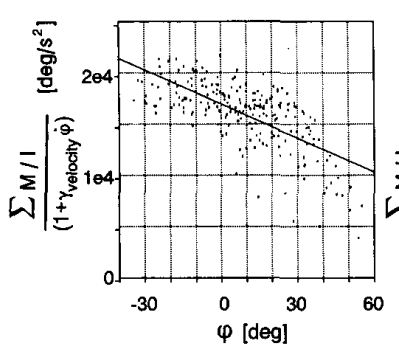

(a)

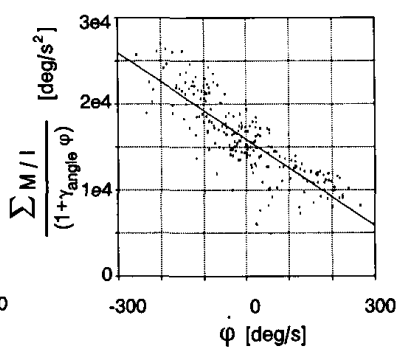

(b)
Fig. 4. (a) Integrated twitch responses compensated for the knee angular velocity dependency $(\Sigma M / I) /\left(1+\gamma_{\text {velocity }} \dot{\varphi}\right)$ versus the corresponding knee angle $\varphi$ taken at $d$ steps after the stimulation pulse. (b) Integrated twitch responses compensated for the knee angle dependency $\Sigma(M / I) /\left(1+\gamma_{\text {angle }} \varphi\right)$ versus the corresponding knee angular velocity $\dot{\varphi}$ taken at $d$ steps after the stimulation pulse (patient TN-left; trial 1). Linear regressions lines are also shown.

than models 1 and 2 for the larger prediction intervals of 500 ms (trial 2) and $1000 \mathrm{~ms}$ (trials 2 and 3) $(\alpha=0.05)$.

The comparable mean values of $J_{\varphi, p}$ for trials 1 and 2 indicate that the identification procedure was consistent. It should also be noted that the performance of trial 3, relative to the rms value of the knee angle in this trial, was not worse than trials 1 and 2, even though the stimulation pulses were applied at half-recruitment and the identification was performed at full recruitment using trial 1 . This is evidence that the recruitment curve actually can be modeled as being independent from the (twitch) dynamics.

\section{Delay}

Identifying the delay $d$ in different regions of the operating range yielded a significant knee angle dependency. It was identified as one time step of $10 \mathrm{~ms}$ smaller in comparison to the estimated delay (Table I) for knee angles $\varphi$ below $-20^{\circ}$ in all subjects, independent of the knee angular velocity. A plausible explanation is that, at short muscle lengths, the muscle must first take up slack before torque can be generated. No significant knee angular velocity dependency showed up. The low resolution in $d$ (units of $T_{s}$ ), however, hampered an accurate estimation of the exact knee angle and knee angular velocity dependency.

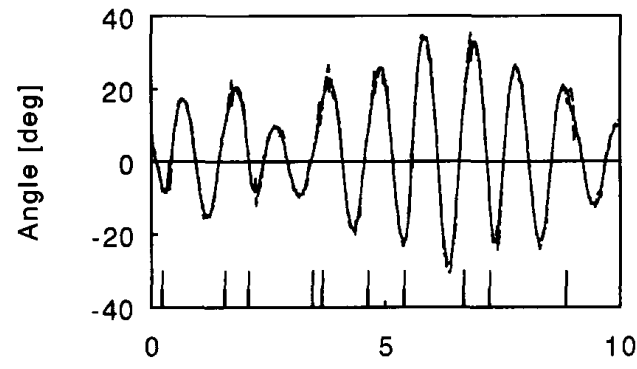

Time $[s]$

(a)

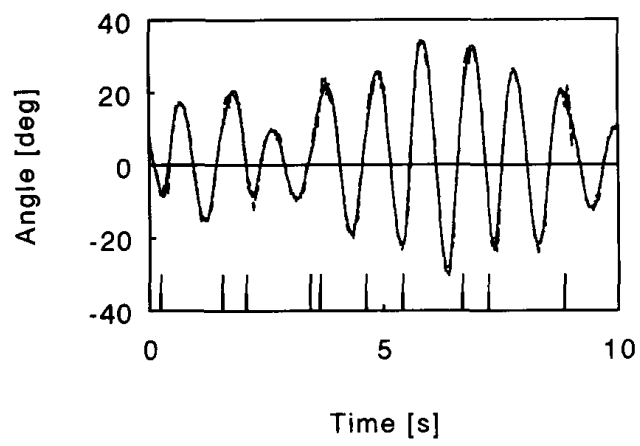

(b)

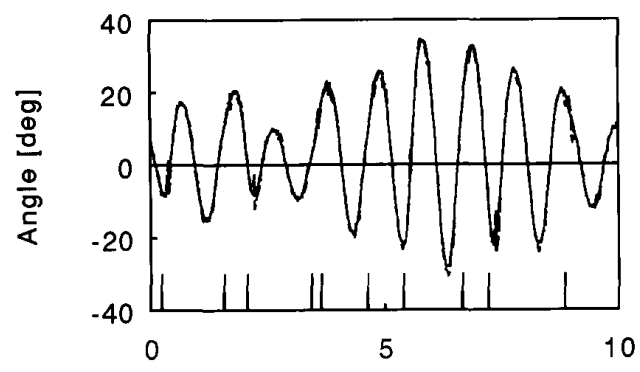

Time [s]

(c)

Fig. 5. Actual (solid) and estimated (dashed) knee angle in subject JM-left. Each point in the graph for the estimated knee angle followed from a prediction ahead for a period of $100 \mathrm{~ms}$ using: (a) model 1, (b) model 2, and (c) model 3.

Identifying the delay using trial 3 , i.e., stimulation pulses at half recruitment, did not reveal a dependency on the recruitment level, values for $d$ being identical to those found in trial 1.

\section{DisCUSSION AND CONCLUSIONS}

The shank system with stimulation of quadriceps, sometimes in combination with hamstrings, has been studied extensively with the main goal to gain insight into the control of electrically stimulated paralyzed human muscle-limb systems [8], [13], [15], [16], [18], [20], [30], [34], [43], [44], [46]. In some of these studies, parametric models were derived. Such models ease the use of computer simulation to investigate 


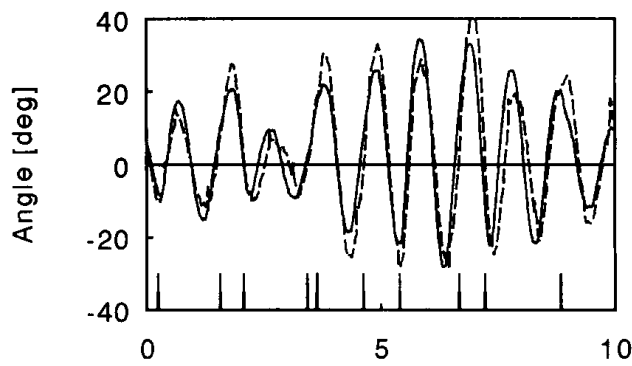

Time [s]

(a)

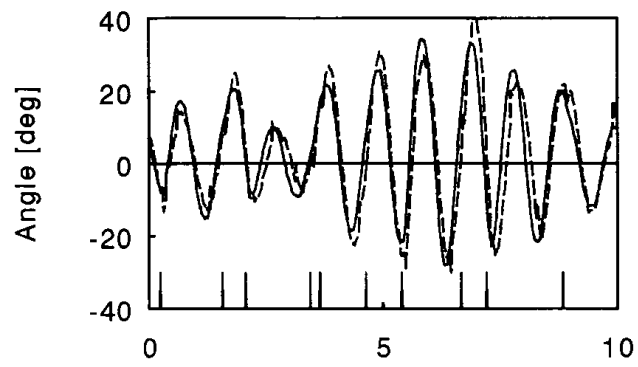

Time [s]

(b)

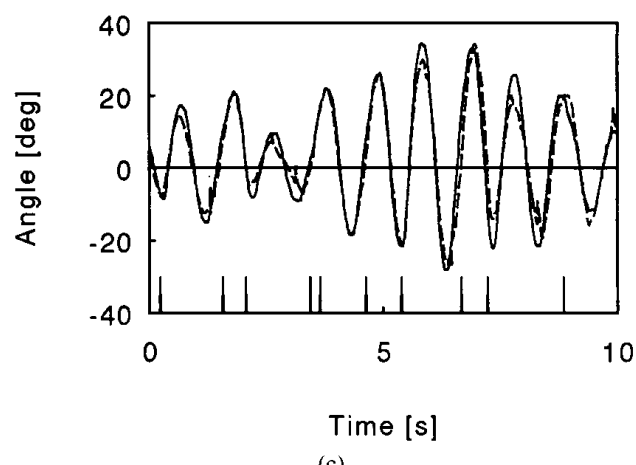

(c)

Fig. 6. Actual (solid) and estimated (dashed) knee angle in subject JM-left Each point in the graph for the estimated knee angle followed from a prediction ahead for a period of $1000 \mathrm{~ms}$ using: (a) model 1, (b) model 2, and (c) model 3.

the performance of control strategies without involving the actual system. This is particularly interesting in rehabilitation engineering since experiments are time-consuming and sometimes burdensome for the participating subjects. Concerning the prediction performance of these parametric models which indicates the validity of its structure and parameters, very little has been documented as yet. Also, the structure of the model required for a given application was not investigated. Parts of models were well-documented, such as isometric recruitment curves [13], [20], isometric torque-knee angle [15], [20], [30], [43], and isokinetic torque-knee angular velocity relations both during tetanic contraction [15], [43], activation dynamics [20], and pendulum descriptions for the shank
TABLE $\mathrm{V}$

Mean Values for the Criterion $J_{\varphi, p}$ (12) for all Participating SUbJects. NOTEWORTHY, ONLY TRIal. 1 Was USED fOR IdENTIFICATION. IN Trials 1 and 2, Stimulation Was applied at Maximal Recruitment. IN Trial 3, Stimulation Was applied at Half-Recruitment. For Each Trial, the Mean Prediction Error with Standard Deviation (S.D. IN Brackets) at Different Prediction Intervals Over All Subjects Is Given Using Models 1, 2, and 3. The Mean rms Value of the Measured KNEE ANGle Was $23.6^{\circ}$ for Trial $1,24.1^{\circ}$ For 2 , and $19.3^{\circ}$ for Trial 3

\begin{tabular}{|c|c|c|c|c|c|c|}
\hline \multirow{2}{*}{$\begin{array}{l}\begin{array}{l}\text { prediction } \\
\text { interval }\end{array} \\
100 \quad[\mathrm{~ms}]\end{array}$} & $\begin{array}{l}\text { mean } \\
\text { model }\end{array}$ & \multicolumn{4}{|c|}{ mean RMS prediction error and s.d. for trial 1 [deg] } & $\begin{array}{l}\text { trial } 1 \text { [deg] } \\
\text { el } 3\end{array}$ \\
\hline & 2.7 & $(0.6)$ & 2.7 & $(0.6)$ & 2.7 & $(0.6)$ \\
\hline $250 \quad[\mathrm{~ms}]$ & 5.7 & $(1.0)$ & 5.8 & $(1.0)$ & 5.4 & (1.1) \\
\hline $500 \quad[\mathrm{~ms}]$ & 6.4 & (1.9) & 6.5 & (1.9) & 5.4 & (1.3) \\
\hline $1000[\mathrm{~ms}]$ & 10.5 & (2.5) & 8.2 & $(2.5)$ & 6.7 & $(1.7)$ \\
\hline $\begin{array}{l}\text { prediction } \\
\text { interval }\end{array}$ & \multicolumn{6}{|c|}{$\begin{array}{l}\text { mean RMS prediction error and s.d. for trial } 2 \text { [deg] } \\
\text { model } 1 \quad \text { model } 2 \quad \text { model } 3\end{array}$} \\
\hline $100[\mathrm{~ms}]$ & 2.5 & $(0.5)$ & 2.5 & $(0.5)$ & 2.5 & $(0.6)$ \\
\hline $250[\mathrm{~ms}]$ & 6.9 & $(1.6)$ & 6.9 & $(1.6)$ & 5.5 & (1.1) \\
\hline $500[\mathrm{~ms}]$ & 9.8 & (3.8) & 9.9 & (3.8) & 6.0 & (1.6) \\
\hline $1000[\mathrm{~ms}]$ & 12.4 & $(4.0)$ & 12.5 & $(4.0)$ & 7.5 & (2.3) \\
\hline $\begin{array}{l}\text { prediction } \\
\text { interval }\end{array}$ & \multicolumn{6}{|c|}{ mean RMS prediction error and s.d. for trial 3 [deg] } \\
\hline 100 [ms] & 1.9 & $(0.4)$ & 1.9 & $(0.4)$ & 1.9 & $(0.4)$ \\
\hline $250[\mathrm{~ms}]$ & 4.6 & $(1.1)$ & 5.2 & $(1.0)$ & 4.5 & (1.1) \\
\hline $500[\mathrm{~ms}]$ & 5.8 & $(1.7)$ & 7.3 & (2.1) & 5.5 & (1.6) \\
\hline $1000[\mathrm{~ms}]$ & 6.7 & $(3.5)$ & 8.2 & (3.5) & 6.3 & (2.4) \\
\hline
\end{tabular}

[8], [16], [41], [44], [46]. Still, the most beneficial use of such models could be expected if they would have predictive capabilities and would have been derived for the total system, including the active and passive subsystems. In the current work, both these passive and active subsystems were modeled and identified during a freely swinging movement from stimulation input to joint movement output [16]. The combined model predicted the knee angle with reasonable accuracy compared to reported errors in model-based knee joint tracking [18], [20], even for the most simple muscle model considered.

The current paper describes the responses to single stimulation pulses. It may allow the prediction of fused responses to stimulation bursts with varying interpulse interval times, considering the responses as a summation of twitch responses as proposed by Bobet et al. [6]. However, this summation is nonlinear [5], [6], [9]. The applicability of the results of this study to fused contractions is still to be evaluated.

One of the conspicuous findings in the current study was the small and comparable prediction error for all model structures in the case of small prediction intervals. A secondorder ARMA model (model 2) performed equally well as the simplest possible model (model 1): A simple delay and gain. Adding angle and angular velocity dependencies (model 3 ) did not show significant improvement. An explanation for these findings is given by the high inertia of the passive system and the low saturation level and significant delay characterizing 
the active system, which results in a dominance of the passive system dynamics at small time intervals. We conclude that for small prediction intervals, a zero-order muscle model with delay is sufficient for the considered movements.

In the design of model-based controllers, model identification and verification should preferably be performed in the range and mode of the desired operation, since the required model structure of the muscle model depends on the load on the muscle [25]-[27], [38], [39]: For example, during the swing phase of gait, the muscle model used for predictive control may be simple and does not require dynamics because of the dominant inertial dynamics of the leg. However, during weight acceptance (load transition) and stance, modeling of the muscle dynamic behavior may be required. In the present paper, we modeled the paralyzed quadriceps-shank system under freely swinging conditions. Accordingly, we used an experimental set-up allowing free movements instead of imposing movements with a dynamometer [40]. A dynamometer may impose torques well outside the range induced by artificially activating muscles. We estimated the torque-angle and torqueangular velocity relations on the basis of the integrated twitch responses. Apparently, the twitches were dependent on the length and shortening velocity of the quadriceps muscle (see Fig 4). In most other studies, torque-angle and torque-angular velocity relations are determined from controlled isometric and isokinetic tetnic contractions, respectively [12], [15], [43]. The torque-angle relation in paralyzed quadriceps muscle then shows a certain maximum at a knee angle of approximately $\varphi=50^{\circ}[15]$, whereas the torque-angular velocity curve in paralyzed quadriceps muscle is hyperbolic according to the well-known Hill relation [15], [21] for concentric contractions and with a plateau for eccentric contractions [15]. In the present study, the maximum of the torque-angle relation was not present in the integrated twitch response (Fig 4(a)). This may be caused by the fact that we considered twitch contractions instead of tetanic contractions.

Various model assumptions could have deteriorated the model performance. Parameters of the shank dynamics (passive system) were assumed to be independent of the active system. Model structures which allow for activation-dependent passive properties might improve the prediction performance but would make on-line identification very difficult. We were particularly interested in a simple model structure with few parameters and acceptable accuracy, suitable for on-line identification and feed-forward or predictive control techniques. Thus, the possible changes in the shank dynamics due to stimulation only occur during stimulation and were assumed to be part of the equivalent acceleration $M / I$ and therefore modeled in the active system. This might have resulted in modeling errors. The shank dynamics were, however, accurately identified for state excitation in the desired operating range [16]. Furthermore, the shank dynamics are relatively slow (dominant) in comparison to the muscle dynamics. Slight changes in the passive system during short muscle contraction will therefore hardly detoriate the model's performance (see Section IV).

The assumption that the knee joint is a perfect one-axial hinge may also have deteriorated model performance. In reality, the nonideal knee joint resulted in changing distance between the accelerometers and the point of rotation, thus resulting in measurement errors (see [50] for a detailed sensitivity analysis). Furthermore, we did not consider the effect of muscle fatigue. It is mainly determined by the number of pulses applied within a certain period of time [7], [15], which was very small in the current study (see, e.g., Fig. 3). Finally, the number of delay steps $d$ was estimated in steps of $T_{s}$, a low resolution, causing slight phase shifts which induced significant prediction errors [16].

One specific measurement error, contributing to possible modeling errors, was caused by the nonrigid attachment of the accelerometers to the shank. Attaching accelerometers to the skin generally results in skin motion artifacts during sudden motions, leading to a resonance error related to the mass of the accelerometers and the compliance of the underlying tissue [35]. In model 2, we tried to model this error with a whitening filter. However, when disturbances on the output are significantly correlated with the excitation input signal, the parameter estimation of this whitening filter becomes troublesome [31]. This might have been the reason why model 2 did not perform better than model 1 . Since models 1 and 3 used the integrated twitch reponse, we assumed that most of the resonance error was averaged out. In any event, it remains questionable whether higher order modeling of muscle activation dynamics ( $A$ in (1)) is worthwhile, considering the dominant inertial properties of the shank.

Parametric models may enable the compensation of disturbances during cyclical movements like gait. Especially during the swing phase of gait, the achievement of objectives like foot clearance, step length, and knee extension at the end of the swing phase [17] can continuously be predicted by the model. This allows for early adaptation of the stimulation during the step, as soon as a disturbance is detected, despite the unfavorable characteristics of the system (high inertia of the leg and low saturation levels of the torques produced by the stimulated muscles). Such disturbance rejection during a cycle can not be achieved by previously reported control methods, like cycle-to-cycle control [17], [44] in which stimulation patterns are open-loop applied during a cycle and adapted between cycles based on an evaluation of the preceding cycles. For this reason, the skeletal-muscle models as studied in this paper can be expected to be of practical use in the control of gait by FES as pursued for rehabilitation of paraplegic patients. However, the model identification may have to be performed at a different joint (hip) and in a different angle range (hips and knees). The system and operating range studied in this paper should be considered as a simple example system, which allows the study of dynamic models and model-based control of which the principles may be applicable in the control of knee and hip movements during gait.

\section{ACKNOWLEDGMENT}

We gratefully acknowledge the researchers of the rehabilitation center 't Roessingh (Enschede, The Netherlands). We also thank the subjects for their participation. 


\section{REFERENCES}

[1] K. J. Åström and B. Wittenmark, Adaptive Control. Reading, MA: Addison-Wesley, 1989

[2] M. L. Audu and D. T. Davy, "The influence of muscle model complexity in musculoskeletal motion modeling," J. Biomed. Eng., vol. 107, pp. $147-157,1985$

[3] R. Baratta, B. H. Zhou, and M. Solomonow, "Frequency response model of skeletal muscle: Effect of pertubation level and control strategy," Med. Biol. Eng. Comput., vol. 27, pp. 337-343, 1989.

[4] P. Bawa, A. Mannard, and R. B. Stein, "Effects of elastic loads in the contraction of cat muscles," Biol. Cyber., vol. 22, pp. 129-137, 1976

[5] L. A. Bernotas, P. E. Crago, and H. J. Chizeck, "A discrete-time model of electrically stimulated muscle," IEEE Trans. Biomed. Eng., vol. BME-33, pp. 829-838, 1986.

[6] J. Bobet, R. B. Stein, and M. N. Oguztöreli, "A linear time-varying model of force generation in skeletal muscle," IEEE Trans. Biomed. Eng., vol. 40, pp. 1000-1006, 1993.

[7] H. B. K. Boom, A. J. Mulder, and P. H. Veltink, "Fatigue during functional neuromuscular stimulation," Progress Brain Res., vol. 97. pp. $409-418,1993$

[8] A. Campisi, P. Baglioni, M. Ferrarin, M. Rabuffetti, and A. Pedotti, "Evaluation of the torque at the knee joint due to electrical stimulation using kinematic analysis," in Ljubljana FES Conference Proceedings, R. Jaeger and T. Bajd, Eds., Aug. 22-25, 1993, pp. 115-118.

[9] S. G. Carroll, R. J. Triolo, H. J. Chizeck, R. Kobetic, and E. B. Marsolais, "Tetanic responses of electrically stimulated paralyzed muscle at varying interpulse intervals," IEEE Trans. Biomed. Eng., vol. 36, pp. $644-653,1989$

[10] T. L. Chia, P. C. Chow, and H. J. Chizeck, "Recursive parameter identification of constrained systems: An application to electrically stimulated muscle," IEEE Trans. Biomed. Eng., vol. 38, pp. 429-442, 1991.

[11] H. J. Chizeck, N. Lang, L. S. Palmiere, and P. E. Crago, "Feedback control of electrically stimulated muscle using simultaneous pulse width and stimulus period modulation," IEEE Trans. Biomed. Eng., vol. 38, pp. 1224-1234, 1991

[12] P. E. Crago, "Muscle input-output model: The static dependency of force on length, recruitment, and firing period," IEEE Trans. Biomed. Eng., vol. 39, pp. 87I-874, 1992.

[13] W. K. Durfee and K. E. Maclean, "Methods for estimating isometric recruitment curves of electrically stimulated muscle," IEEE Trans. Biomed. Eng., vol. 36, pp. 654-667, 1989.

[14] B. Flaherty, C. J. Robinson, and G. C. Agarwal, "Determining appropriate models for joint control using surface electrical stimulation of soleus in spinal cord injury," Med. Biol. Eng. Comput., vol. 32, pp. 273-282, 1994

[15] H. M. Franken, P. H. Veltink, M. Fidder, and H. B. K. Boom, "Fatigue of intermittently stimulated paralyzed human quadriceps during imposed cyclical lower leg movements," J. Electromyogr. Kinesiol., vol. 3, pp. 3-12, 1993

[16] H. M. Franken, P. H. Veltink, R. Tijsmans, H. Nijmeijer, and H. B. $\mathrm{K}$. Boom, "Identification of passive knee joint and shank dynamics in paraplegics using quadriceps stimulation," Trans. Rehab. Eng., vol. 1, no. 3, pp. 154-164, Sept. 1993.

[17] H. M. Franken, P. H. Veltink, G. Baardman, R. A. Redmeijer, and H. B. $\mathrm{K}$. Boom, "Cycle-to-cycle control of the swing phase of paraplegic gait induced by funtional electrical stimulation," Med. Biol. Eng. Comput., in press.

[18] M. S. Hatwell, B. P. Oderkerk, C. A. Sacher, and G. F. Inbar, "The development of a model reference adaptive controller to control the knee joint of paraplegics," IEEE Trans. Biomed. Eng., vol. 36, pp. 683-691, 1991.

[19] H. Hatze, "A complete set of control equations of the human musculoskeletal system," J. Biomechan., vol. 10, pp. 799-807, 1977.

[20] J. M. Hausdorff and W. K. Durfee, "Open-loop position control of the knee joint using electrical stimulation of the quadricpes and hamstrings," Med. Biol. Eng. Comput., vol. 29, pp. 269-280, 1991

[21] A. V. Hill, "The heat of shortening and the dynamic constants of muscle," in Proc. Roy. Soc., London, vol. B126, pp. 136-195, 1938.

[22] I. W. Hunter and M. J. Korenberg. "Generation of random sequences with jointly specified probability density function and autocorrelation functions," Biol. Cyber., vol. 47, pp. 141-146, 1983

[23] _ , "The identification of nonlinear biological systems: Wiener and Hammerstein cascade models," Biol. Cyber., vol. 55, pp. 135-144, 1986.

[24] A. F. Huxley, "Muscle structures and theories of contraction," Prog. Biophys. Biophys. Chem., vol. 7, pp. 257-318, 1957.
[25] G. C. Joyce, P. M. H. Rack, and D. R. Westbury, "The mechanical properties of cat soleus muscle during controlled lengthening and shortening movements," J. Physiol., vol. 204, pp. 46l-474, 1969.

[26] G. C. Joyce and P. M. H. Rack, "Isotonic lengthening and shortening movements of the cat soleus muscle," J. Physiol., vol. 204, pp. 475-491, 1969.

[27] B. Katz, "The relation between force and speed in muscular contraction," J. Physiol., vol. 96, pp. 45-64, 1939.

[28] N. Lan, P. E. Crago, and H. J. Chizeck, "Feedback control methods for task regulation by electrical stimulation of muscles," IEEE Trans. Biomed. Eng., vol. 38, no. 12, pp. 1213-1223, 1991.

[29] M. Lemay, P. E. Crago, M. Katorgi, and G. J. Chapman, "Automated tuning of a closed-loop hand grasp neuroprosthesis," IEEE Trans. Biomed. Eng., vol. 40, pp. 675-685, 1993.

[30] M. Levy, J. Mizrahi, and Z. Susak, "Recruitment, force and fatigue characteristics of quadriceps muscles of paraplegics isometrically activated by surface functional electrical stimulation," J. Biomed. Eng., vol. 12, pp. $150-156,1990$.

[31] L. Ljung, System Identification, Theory for the User. Englewood Cliffs, NJ: Prentice-Hall, 1987.

[32] A. Mannard and R. B. Stein, "Determination of the frequency response of isometric soleus muscle in the cat using random nerve stimulation," J. Physiol., vol. 229, pp. 275-281, 1973.

[33] E. B. Marsolais and R. Kobetic, "Functional electrical stimulation for walking in paraplegia," J. Bone Joint Surg., vol. 69-A, pp. 728-733, 1987

[34] D. R. McNeal, R. J. Nakai, P. Meadows, and W. Tu, "Open-loop control of the freely-swinging paralyzed leg," IEEE Trans. Biomed. Eng., vol. 36, pp. 895-905, 1989

[35] L. Nokes, J. A. Fairclough, W. J. Mintowt-Czyz, and J. Williams, "Vibration analysis of the human tibia: The effect of soft tissue on the output from skin-mounted accelerometers," J. Biomed. Eng., vol. 6 , pp. 223-226, 1984

[36] J. S. Petrofsky and C. A. Phillips, "The influence of recruitment order and fiber composition on the force-velocity relation and fatiguability of skeletal muscles in the cat," Med. Biol. Eng. Comput., vol. 18, pp. $381-390,1980$

[37] A. Prochazka, "Comparison of natural and artificial control of movement," IEEE Trans. Rehab. Eng., vol. 1, pp. 7-17, 1993.

[38] P. M. H. Rack, "The behavior of mammalian muscle during sinussoidal stretching," J. Physiol., vol. 183, pp. 1-15, 1966

[39] P. M. H. Rack and D. R. Westbury, "The effect of length and stimulus rate on tension in the isometric soleus muscle," J. Physiol., vol. 204, pp. $443-460,1969$

[40] C. J. Robinson, B. Flaherty, L. Fehr, G. C. Agarwal, G. F. Harris, and G. L. Gottlieb, "Biomechanical and reflex responses to joint perturbations during electrical stimulation of muscle: Instrumentation and measurement techniques," Med. Biol. Eng. Comput., vol. 32, pp. 261-272, 1994

[41] A. Scheiner, R. B. Stein, D. C. Ferencz, and H. J. Chizeck, "Improved models for the lower leg in paraplegics," in 15 th Int. Conf. IEEE Eng. Med. Biol. Soc., Y. J. Szeto and R. M. Rangayyan, Eds., San Diego, CA, 1993, pp. 1151-1152.

[42] G. H. Shue, P. E. Crago, and H. J. Chizeck, "Muscle joint models incorporating activation dynamics, torque-angle and torque-velocity properties," IEEE Trans. Biomed. Eng., vol. 42, pp. 212-223, 1995

[43] R. J. Triolo, D. E. Robinson, and R. R. Betz, "Force-velocity and length-tension properties of stimulated human quadriceps in spinal cord injured children," in Ilth Int. Conf. IEEE Eng. Med. Biol. Soc., 1989. pp. $967-968$

[44] P. H. Veltink, "Control of FES-induced cyclical leg movements of the lower leg," Med. Biol. Eng. Comput., vol. 29, pp. NS8-NS12, 1991.

[45] P. H. Veltink, H. J. Chizeck, P. E. Crago, and A. El-Bialy, "Nonlinear joint control for artificially stimulated muscle," IEEE Trans. Biomed. Eng., vol. 39, pp. 368-380, 1992

[46] P. H. Veltink, R. Tijsmans, H. M. Franken, and H. B. K. Boom, "Identification of electrically stimulated quadriceps-Lower leg dynamics-The use of accelerometers for estimating knee joint acceleration and quadriceps torque," in Proc. 14th Int. Conf. IEEE Eng. Med. Biol. Soc., Paris, 1992, pp. 1339-1340.

[47] J. M. Winters and L. Stark, "Analysis of fundamental human movement patterns through the use of in-depth antagonist muscle models," IEEE Trans. Biomed. Eng., vol. BME-32, no. 10, pp. 826-839, 1985.

[48] _ _ "Muscle models: What is gained and what is lost by varying muscle model complexity," Biol. Cyber., vol. 55, pp. 403-420, 1987.

[49] D. R. Wilkie, "Relation between force and velocity in human muscle," J. Physiol., vol. 110, pp. 249-280, 1950. 
[50] A. T. M. Willemsen, C. Frigo, and H. B. K. Boom, "Lower extremity angle measurement with accelerometers-Error and sensitivity analysis," IEEE Trans. Biomed. Eng., vol. 38, pp. 1186-1193, 1991.

[51] G. I. Zahalak, "A distribution-moment approximation for kinetic theories of muscular contraction," Math. Biosci., vol. 55, pp. 89-114, 1981.

[52] F. E. Zajac, "Muscle and tendon: Properties, models, scaling, and application to biomechanics and motor control," Crit. Rev. Biomed. Eng., vol. 17 , pp. $359-410,1989$.

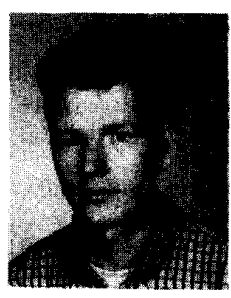

Henry M. Franken (S'94) received the M.Sc. degree in electrical engineering from the University of Twente, Enschede, The Netherlands, in 1990 (topic: Cartesian control of a six-link robot). He received the Ph.D. degree cum laude (topic: control system design for FES-assisted paraplegic gait) at the Institute of Biomedical Technology (BMTI) of the University of Twente.

His research interests involve applications of systems engineering. He is currently a member of the scientific staff of the Telematics Research Center (TRC), The Netherlands.

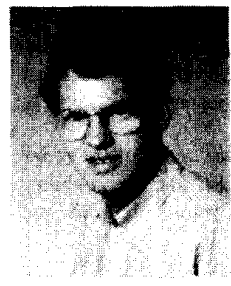

Peter H. Veltink (S'85-M'88) received the M.Sc. degree in electrical engineering from the University of Twente, Enschede, The Netherlands, in 1984 and the Ph.D. degree in 1988 (topic: recruitment of myelinated nerve fibers during artificial nerve stimulation).

In 1989, he was a Visiting Assistant Professor at Case Western Reserve University, Cleveland, $\mathrm{OH}$, where he did research in the area of nonlinear control of muscle contraction. Currently, he is a faculty member of the Biomedical Engineering Department of the Faculty of Electrical Engineering, University of Twente. His research is in the area of control of functional neuromuscular stimulation and the application of body mounted sensors for human movement analysis. In this rehabilitation technology research, he works closely together with the Research Department of the Roessingh Rehabilitation Center in Enschede.

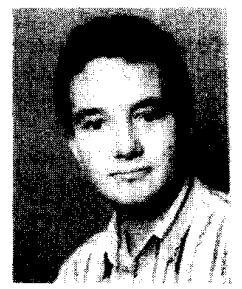

Roel Tijsmans received the M.Sc. degree in electrical engineering from the University of Twente, Enschede, The Netherlands, in 1993. During his study, he spent three months at Strathclyde University (Glasgow, Scotland) working on FES controlled standing of paraplegics. His final project was on the modeling and identification of artificially stimulated muscle during dynamic contraction. Currently, he is following a two-year designer course in mechatronics.

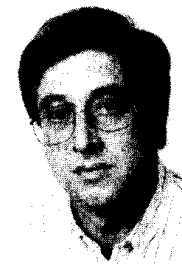

Henk Nijmeijer (M'83-SM'91) received both the university degree and doctorate in mathematics from the University of Groningen. The Netherlands, in 1979 and 1983, respectively.

After spending three years at the Center for Mathematics and Computer Science (CWI) in Amsterdam, he moved to the Department of Applied Mathematics of the Twente University of Technology in 1983, where he is presently an Associate Professor (UHD). His main research interests are in the area of nonlinear and linear geometric control theory and its applications. He is an author (with A. J. van der Schaft) of Nonlinear Dynamical Control Systems (New York: Springer-Verlag, 1990).

Dr. Nijmeijer is a member of the editorial board of the SIAM Joumal on Control and Optimization, the IFAC Journal Automatica, the International Journal of Robust and Nonlinear Control, and the Journal of Dynamical and Control Systems.

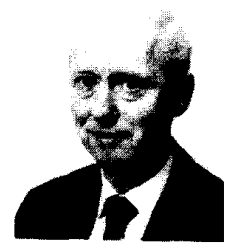

Herman B. K. Boom was born in 1933 and has been trained as a Medical Physicist at the University of Utrecht, The Netherlands. He received the Ph.D. (cum laude) in 1971 and conferred on a dissertation on ventricular mechanics.

He then joined the scientific staff of the Department of Medical Physiology of the University of Utrecht, where he was engaged in research on ventricular dynamics, and where he taught biophysics and physiology to medical students. Since 1976, he has been a Professor of Medical Electronics at the University of Twente, Enschede, The Netherlands, teaching electromagnetism and bioelectricity to electrical engineering students. He is directing research as Chairman of the Department of Medical Information Technology of the University of Twente, which enrolls seven faculty members. The department is part of the faculty of electrical engineering and is also integrally contributing to the university's research institutes for Biomedical Technology (BMTI) and Microelectronics, Sensors, and Actuators (MESA). He chairs the Center for Rehabilitation Technology (CERT), which is a formal cooperation between the University of Twente and the Roessingh Rehabilitation Center, Enschede, The Netherlands. In this center, more than 40 members of staff are participating in research on rehabilitation medicine and rehabilitation technology. He has authored and coauthored papers on cardiovascular physiology, biophysics and technology, bioelectricity, muscle dynamics and rehabilitation engineering, which are also the current fields of his research interests.

Dr. Boom has been active in the organization of international conferences, including the Annual International Conferences of the Engineering in Medicine and Biology Society. He will be the Conference Chair for this conference's 1996 meeting to be held in Amsterdam. The Netherlands. He has served as an international referee for many submitted papers and grant proposals, as well as in various committees for the evaluation of academic chairs and programs. 